This manuscript is a preprint and has not been submitted for traditional publication yet, we have a few options in mind but have not formally submitted a manuscript. Please note that this article has not undergone peer-review and the manuscript has yet to be formally accepted for publication. Please feel free to contact any of the authors; we welcome feedback.

Example Citation: D. Huang, F. Liu, Seiching period from experiment and numerical simulation, 2021. 


\title{
Seiching period from experiment and numerical simulation
}

\author{
David Huang, Milbourne High School, NJ 07041 USA, \\ Fei Liu, New Jersey Science Academy, NJ 08807 USA Fei.Liu@njsci.org
}

October 18, 2021

\begin{abstract}
Seiching is a common phenomenon in nature where a large body of water exhibits periodic behavior. First, we set up water oscillations in a tank and measure its oscillation period. Then we use OpenFOAM to simulate the oscillation behavior of the water waves in a $3 \mathrm{D}$ mesh with the same dimensions as the tank used in the experiment. Applying the Fourier transformation on the velocities calculated from OpenFOAM, we identify the period of the fundamental mode of oscillation. The result from the measurements and the simulations are presented and then compared with solutions from linearized shallow water equations. The experimental measurements, numerical simulations and SWE solutions show good agreement.
\end{abstract}

Keywords: Seiching, OpenFOAM, Shallow water equation, Oscillation, Wave

\section{Introduction}

A seiche is a standing wave oscillating in an enclosed or partially enclosed body of water. Such phenomena are commonly observed in lakes, bays, harbours, and reservoirs. The first scientific observation and documentation of seiching was performed in Lake Geneva [1. Seiching in harbours often exhibits nonlinear interaction between gravity waves and wind generated capillary waves [2].

Seiching in lakes or reservoirs may be difficult to notice due to the long oscillation period resulting from the long wave length and the slow wave speed. The period of seiching waves can be estimated from Merian's formula [3],

$$
T=\frac{2 L}{\sqrt{g h}}
$$

where $T$ is the longest natural period, and $L$ and $h$ are the width and height of the body of water respectively.

The effect of seiching in lakes and harbours is similar to the effect of storm surges where boats can be sent to the docks and shores due to the sudden onset of water oscillation [4]. Most natural seichings in lakes and reservoirs are triggered by earthquakes [5]. In the ocean and seas, seiching waves can be generated from earthquakes, tides, and tsunamis [6].

With the advance of computational fluid dynamics (CFD) simulations, seiching can be modelled relatively easily on computers. Past work show a range of interest from modelling the fundamental modes of seiching oscillations [7] [8] [9] to further investigation of complex wave behavior due to non-linear interactions [10] [11.

In this study we compare observations, simulations and theoretical predictions for seiching 
in a relatively simple geometry. In the following sections, we describe the results from each approach, mainly focusing on comparing the period of oscillation obtained from the three different approaches.

\section{Experiment Setup}

For the seiching experiment, the following tools and materials are used: a water container, tap water, a smart phone as a stopwatch, and a Google spreadsheet to record data. Water is prepared at the specific depth before each measurement (Figure 1). The following water depths for the container are measured: $1 \mathrm{~cm}, 1.5 \mathrm{~cm}, 2 \mathrm{~cm}$, $2.5 \mathrm{~cm}, 3 \mathrm{~cm}, 4 \mathrm{~cm}, 5 \mathrm{~cm}, 6 \mathrm{~cm}, 8 \mathrm{~cm}, 9 \mathrm{~cm}$, and $10 \mathrm{~cm}$ as shown in column 1 Table 1, Ten trials are performed for each depth, and the average period measured for that particular depth is recorded in column 2 (Measurement). The following details describe how the average period is measured.

In order to obtain the oscillations, the container is shaken left and right gently, and after two full periods (which consists of the water oscillating from one side of the container to the other side), the timer on the smart phone is started. Once the oscillations become less obvious, the timer is stopped and the data is compiled onto the spreadsheet. Measurement of the period for each depth is repeated for about 10 times to reduce measurement error. After the measurements are obtained, the average oscillation period from the repeated measurements is calculated for each depth, along with the standard deviation (column 3 in Table 1).

Through the power function regression for the measured period $T$ and depth $h$, we obtain the

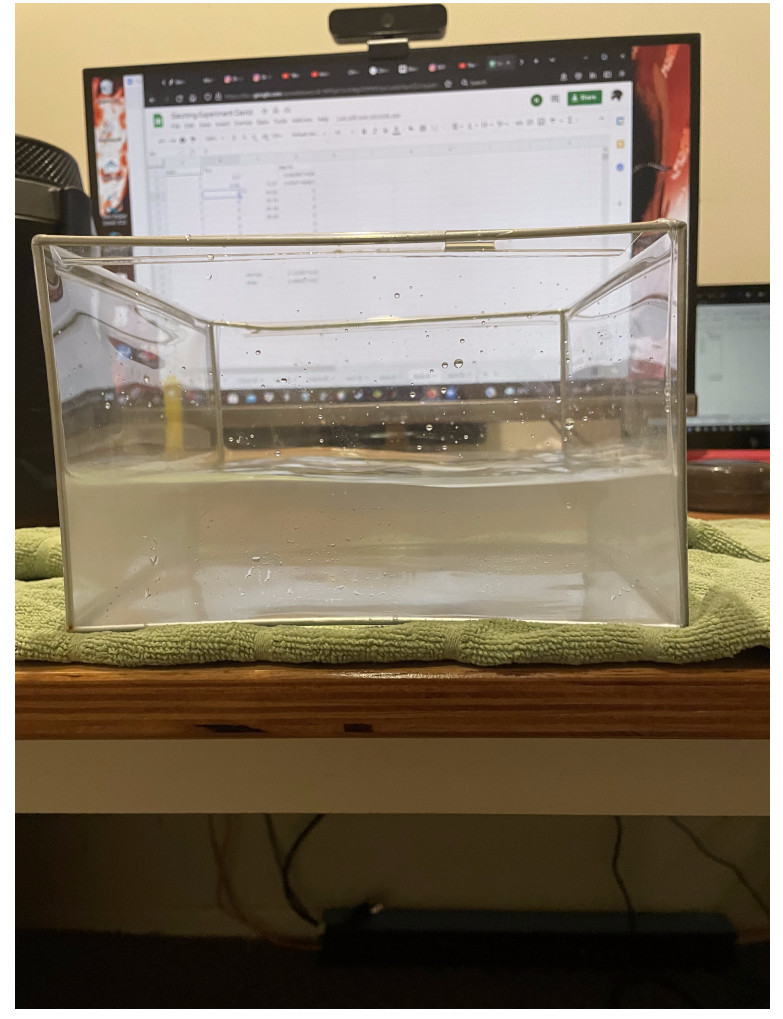

Figure 1: Water tank used in the experimental setup.

following empirical relationship:

$$
T=1.25 * h^{-0.396}
$$

A set of predicted period based on the regression equation 2 is recorded in column 4 (Predicted) on Table 1 .

\section{Simulation Setup}

OpenFOAM is a popular open source CFD package that supports the finite volume [12] approach to fluid dynamics simulations. In addition, the finite volume of the mesh [13] is adaptive and can be refined near the surface of water 


\begin{tabular}{|c|c|c|c|c|c|}
\hline Depth & Measurement & stdev & Predicted & Simulated & SWE \\
\hline 1 & 1.25 & 0.0631 & 1.25 & & 1.24 \\
\hline 1.5 & 1.05 & 0.0421 & 1.07 & 1.09 & 1.01 \\
\hline 2 & 0.933 & 0.0305 & 0.952 & 0.900 & 0.876 \\
\hline 2.5 & 0.793 & 0.0141 & 0.871 & 0.800 & 0.784 \\
\hline 3 & 0.735 & 0.0347 & 0.810 & 0.750 & 0.716 \\
\hline 4 & 0.702 & 0.0456 & 0.723 & 0.660 & 0.620 \\
\hline 5 & 0.662 & 0.0252 & 0.662 & 0.610 & 0.554 \\
\hline 6 & 0.642 & 0.0193 & 0.615 & 0.575 & 0.506 \\
\hline 7 & 0.636 & 0.0488 & 0.580 & 0.560 & 0.468 \\
\hline 8 & 0.528 & 0.0213 & 0.550 & 0.540 & 0.438 \\
\hline 9 & 0.583 & 0.0108 & 0.525 & 0.540 & 0.413 \\
\hline 10 & 0.554 & 0.0177 & 0.503 & 0.530 & 0.392 \\
\hline
\end{tabular}

Table 1: The seiching period (in seconds) from the measurements, the simulation, and the theoretical SWE formula as a function of depth (in centimeters).

to capture the details of the flow field near the water air interface. The following subsections discusses how OpenFOAM is set up to perform the numerical simulation of the seiching in the water tank.

\subsection{Geometry and Initial condition}

The rectangular water tank (Figure 1) used in both the experiment and the simulation has an inner dimension of $0.194 \mathrm{~m}$ in width, $0.194 \mathrm{~m}$ in length, and $0.15 \mathrm{~m}$ in height. An identical geometry is used in the numerical simulation.

In order to initiate the water wave in the tank, the initial height of water is set in a staggered pattern (Figure 2) based on the mean depth of water $d, d+d / 4, d+d * 3 / 16, d+d / 8, d+d / 16, d-$ $d / 16, d-d / 8, d-d * 3 / 16, d-d / 4$. This uneven initial height allows water oscillation to develop in the numerical simulation.

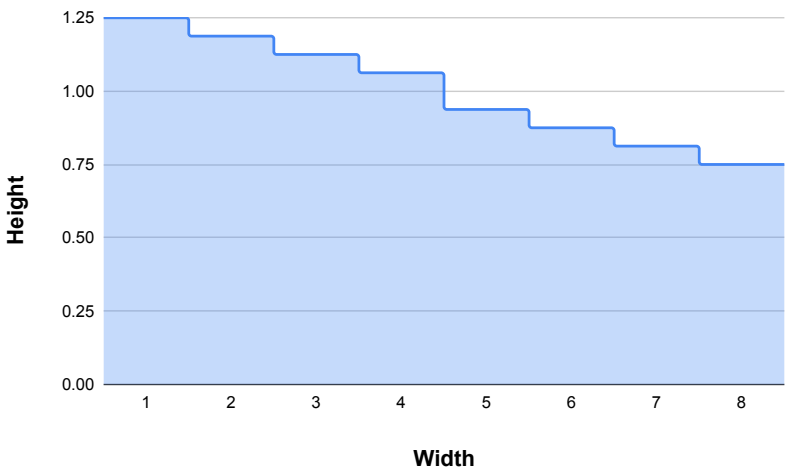

Figure 2: The initial volume of water in the tank. The total volume and average depth can be easily calculated from such a set up. The uneven height as a function of position along the width allows the seiching wave to develop naturally.

\subsection{Meshing}

In addition to a uniform coarse resolution mesh generated for the entire space within the tank, a 
refinement mesh is used near the water surface to capture the details of the water wave (Figure 3). The resolution of the coarse resolution mesh is $9.7 * 10^{-3} \mathrm{~m}$ in width and $9.7 * 10^{-3} \mathrm{~m}$ in length. A resolution that depends on the depth $d$ of water is used in the simulation $\frac{2 * d}{30}$ in the vertical direction.

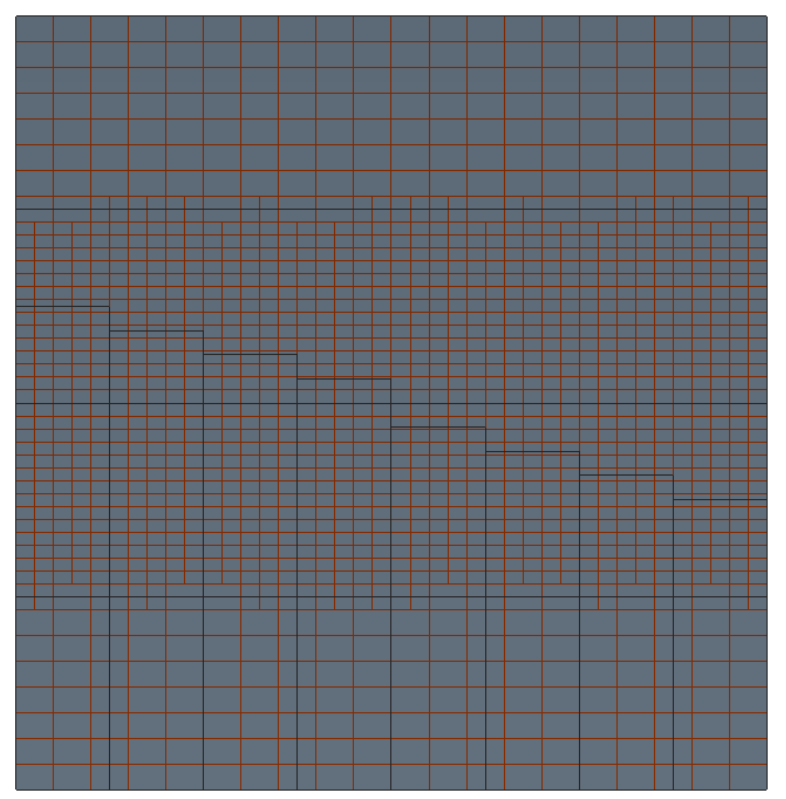

$$
\begin{array}{r}
\nabla \cdot \vec{v}=0 \\
\frac{\partial \vec{v}}{\partial t}+\nabla \cdot(\vec{v} \otimes \vec{v})=-\nabla p+\nabla(\nu \nabla \vec{v})
\end{array}
$$

The pressure-velocity coupled equation is solved by decoupling the pressure and momentum fields through predictor-corrector steps. In this particular simulation, the $k-\epsilon$ [16] turbulence mode is enabled. The Reynolds number $R e=\frac{v L}{\nu}$ far exceeds the laminar regime of water flow due to the small dynamic viscosity $\nu$ of water.

\subsection{Result}

Simulated water level changes periodically in the water tank. Figure 4 shows the water level at 0.5 seconds after the model has started simulation for an average water depth of $10 \mathrm{~cm}$. Figure 5 shows the velocity of a monitored point in the water tank as a function of simulation time. The periodic behavior of the velocity as a function of time is apparent. Non-uniform Fourier analysis (Figure 6) is applied on the velocity time data to calculate the oscillation period shown in column

Figure 3: The mesh of the water tank. The air water interface has a higher resolution.

\subsection{Solver}

OpenFOAM Inter solver is a multi phase NavierStokes equations solver based on the finite volume [12] approach to solve the incompressible viscous fluid flow [14] [15]. The governing equations of the incompressible viscous fluids are the typical incompressible unsteady NavierStokes equations:

Note that OpenFOAM is not able to correctly simulate the seiching oscillation at a depth of 1 $\mathrm{cm}$. The cell for the simulated $1 \mathrm{~cm}$ depth is left blank in Table 1. The numerical simulation is unable to converge despite the use of dynamic time stepping.

\section{Theoretical Consideration}

The period of seiching can be derived for simple geometry and small amplitude oscillation using the shallow water equations with suitable approximations. 


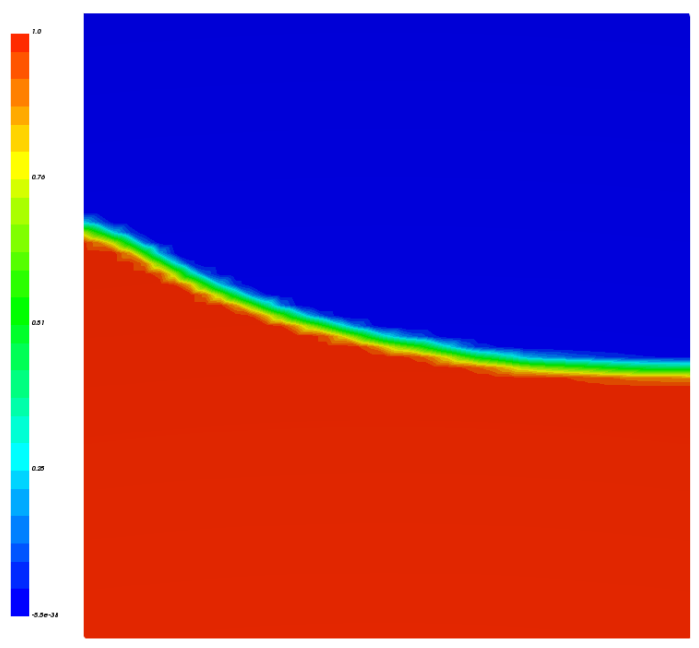

Figure 4: The simulated water level at $0.5 \mathrm{~s}$ after the water begins oscillating. The red is water, the blue is air, and the intermediate colors represent the air water interface. The initial average water height is $10 \mathrm{~cm}$.

The complete incompressible shallow water equation for the dependence of height $h$, speed $u$ and $v$ on time $t$

$$
\begin{array}{r}
\frac{\partial h}{\partial t}+\frac{\partial h u}{\partial x}+\frac{\partial h v}{\partial y}=0 \\
\frac{\partial u}{\partial t}+u \frac{\partial u}{\partial x}+v \frac{\partial u}{\partial y}+g \frac{\partial h}{\partial x} \\
-\frac{\nu}{h}\left(2 \frac{\partial^{2} h u}{\partial x^{2}}+\frac{\partial^{2} h u}{\partial x^{2}}+\frac{\partial^{2} h v}{\partial x \partial y}\right)=0 \\
\frac{\partial v}{\partial t}+u \frac{\partial v}{\partial x}+v \frac{\partial v}{\partial y}+g \frac{\partial h}{\partial y} \\
-\frac{\nu}{h}\left(\frac{\partial^{2} h v}{\partial x^{2}}+2 \frac{\partial^{2} h v}{\partial y^{2}}+\frac{\partial^{2} h u}{\partial x \partial y}\right)=0
\end{array}
$$

In the limit where $\nu \approx 0, u \ll \sqrt{g h}$ and $v \ll$ $\sqrt{g h}$, let $h=H+\delta h$ where $H$ is the mean depth of water and $\delta h$ is the height variation from the

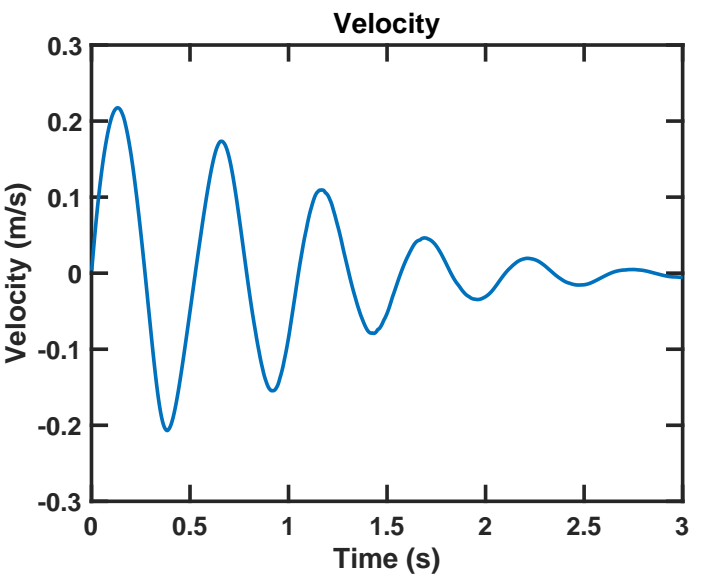

Figure 5: The velocity $(\mathrm{m} / \mathrm{s})$ component of a point within the water simulated as a function of time. This velocity component $U_{x}$ is along the direction of the length of the water tank. The velocity time graph exhibits clear periodic behavior. The initial average water height is 10 $\mathrm{cm}$.

mean depth $\delta h \ll H$, it can be shown the above equations can be linearized into

$$
\frac{\partial^{2} h}{\partial t^{2}}=g H \frac{\partial^{2} h}{\partial x^{2}}+g H \frac{\partial^{2} h}{\partial y^{2}}=g H \nabla^{2} h
$$

The wave speed from the linearized shallow water equation is therefore $c=\sqrt{g H}$. The momentum terms when $u \sim c$ or $v \sim c$ can affect the oscillation periods through non-linear interaction.

When the fundamental mode of a standing seiching wave has a wavelength that is twice the length of the domain where the middle of the tank is a node, the period is identical to Merian's formulation:

$$
T=\frac{2 L}{\sqrt{g H}}
$$

The numbers based on this formulation are recorded in column 6 (SWE) on Table 1 . 


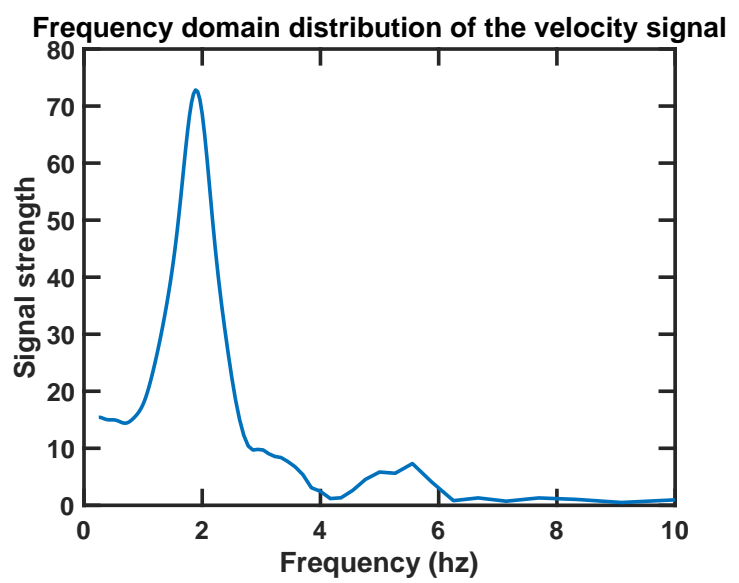

Figure 6: Frequency analysis of the velocity calculated. It can be seen clearly there is a dominant mode (about $2 \mathrm{hz}$ or a period of $0.530 \mathrm{sec}-$ ond) of oscillation for the water motion. The initial average water height is $10 \mathrm{~cm}$.

\section{Conclusion and future work}

The seiching period from the measurements, the simulation, and the theory are summarized in the Table11. Figure 7 shows all the results including the 'Predicted' column using the regressed empirical equation 2.

We can see that measurement and simulation show remarkable agreement which is a testament of the power of computational fluid dynamics. The results using the derived equation based on the linearized shallow water equation 9 agree well when $\frac{h}{L} \leq 0.05$. As the depth of the water increases, the discrepancy widens where the nonlinear effects from the shallow water equation become important when the water is no longer shallow.

While OpenFOAM is unable to simulate the oscillation at $1 \mathrm{~cm}$ depth, both the measurements and the theoretical consideration show close agreement at 1.25 seconds for this depth. This is a depth at which SWE approximation works well. We can see this close agreement from Figure 7 at the $1 \mathrm{~cm}$ depth.

The CFD approach laid out in this work can be applied to simulating seiching oscillations in lakes, harbors, and bays [17] [18] [19] based on the 3 dimensional elevation data of the bottom terrain. It'll be interesting to set up a comparison between observed seiching period and numerical simulation for one of the lakes or harbors in the US.

Some additional interesting topics include numerical simulation of a seiching oscillation driven by wind [20] and investigation of the more complex non-linear interaction between wind waves and gravity waves. [21] [22] [23].

\section{References}

[1] Darwin, G. H. (1898). The Tides and Kindred Phenomena in the Solar System. London: John Murray. pp. $21-31$.

[2] Munk, Walter H. (1950). Origin and generation of waves. 1st International Conference on Coastal Engineering, Long Beach, California. Council on Wave Research, American Society of Civil Engineers. doi:10.9753/icce.v1.1. ISSN 2156-1028.

[3] Proudman, J. (1953). Dynamical oceanography. London: Methuen. $\S 117$ (p. 225). OCLC 223124129.

[4] Korgen, Ben (February 2000). "Bonanza for Lake Superior: Seiches Do More Than Move Water". seagrant.umn.edu. University of Minnesota Duluth. Archived from the original on 2007-12-27.

[5] Arizona Geology: Video of seiche in Devils Hole pupfish pond. (Posted: April 27, 2010). 2010-04-27. Retrieved 17 October 2014.

[6] Chapman, David C.; Graham S. Giese (1990). "A Model for the Generation of Coastal Seiches by Deep-Sea Internal Waves". J. 


\section{Period of Seiching Oscillation}

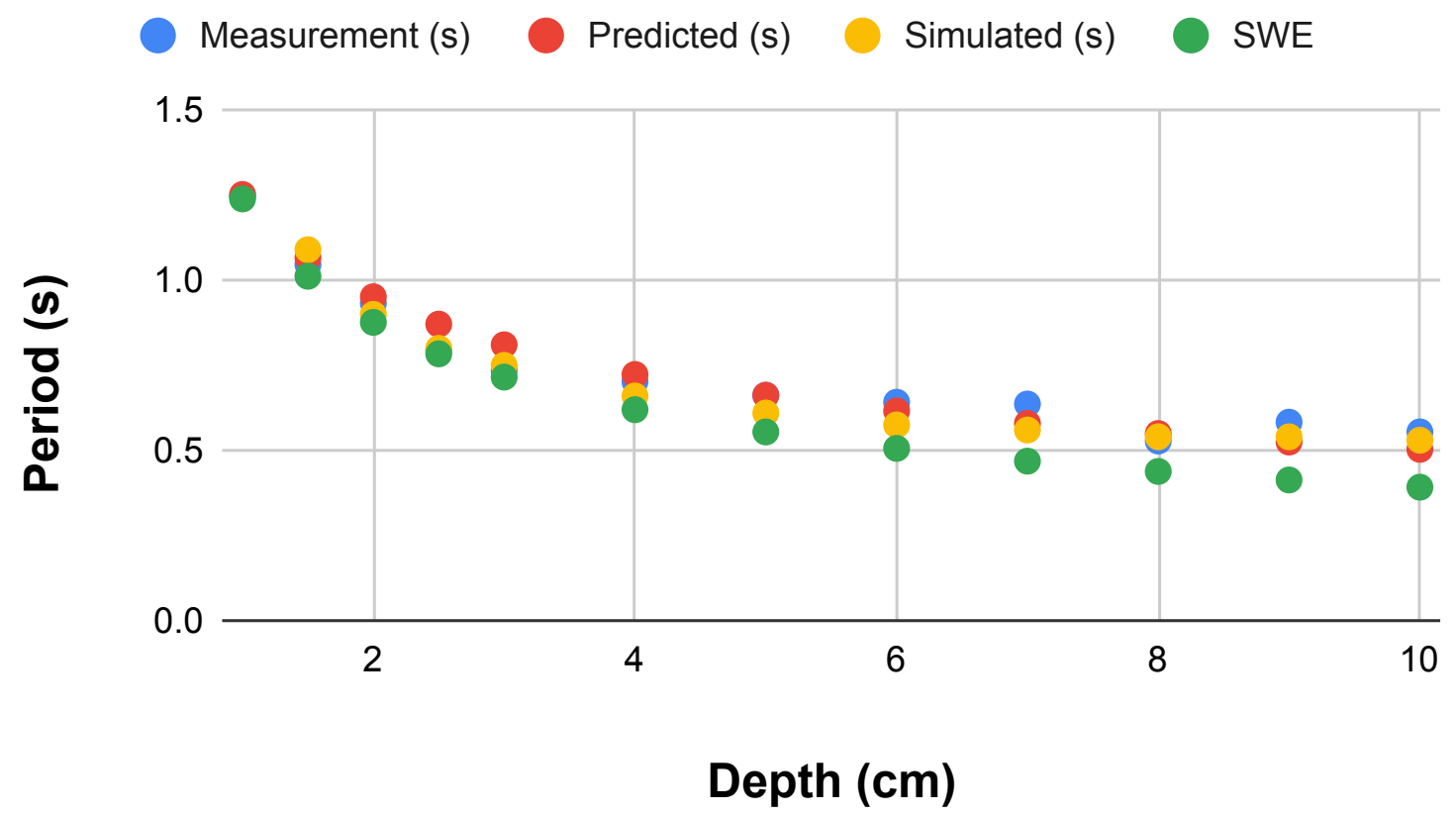

Figure 7: The period of water oscillation based on measurement (blue), predicted using the regression equation 2(red), the simulation (yellow), and the shallow water equation (SWE, green). Measurements, Simulated, and SWE solutions show the same trend in the resulting seiching period.

Phys. Oceanogr. 20 (9): 1459-1467. Bibcode:1990JPO...20.1459C. doi:10.1175/15200485(1990)020;1459:AMFTGO ¿2.0.CO;2.

[7] Manilyuk, Y.V., Cherkesov, L.V. Simulation of seiche oscillations in the Sea of Azov, using the finite element technique. Phys. Oceanogr. 6, 325-329 (1995). https://doi.org/10.1007/BF02197480

[8] Abbas Dorostkar, Leon Boegman, Andrew Pollard, Three-dimensional simulation of highfrequency nonlinear internal wave dynamics in Cayuga Lake Journal of Geographical Research, Volume 122, Issue 3 March 2017. https://doi.org/10.1002/2016JC011862Citations: 14
[9] Canitano Alexandre, Bernard Pascal, Allgeyer Sébastien, Observation and modeling of the seismic seiches triggered in the Gulf of Corinth (Greece) by the $2011 \mathrm{Mw} 9.0$ Tohoku earthquake, Journal of Geodynamics, Volume 109, p. 24-31, September 2017. DOI: $10.1016 /$ j.jog.2017.06.001

[10] Aiki, H., J. P. Matthews, and K. G. Lamb (2011), Modeling and energetics of tidally generated wave trains in the Lombok Strait: Impact of the Indonesian throughflow, J. Geophys. Res., 116, C03023, doi:10.1029/2010JC006589.

[11] Arthur, R. S., and O. B. Fringer (2014), The dynamics of breaking internal solitary waves on slopes, J. Fluid Mech., 761, 360- 398, doi:10.1017/jfm.2014.641. 
[12] Jasak H., 1996, "Error Analysis and Estimation for the Finite Volume Method with applications to Fluid Flows", Direct, M

[13] Owen, S. J. 1998. A survey of unstructured mesh generation technology. In Proc. 7th Inter. Mesh. Roundtable, 239-267.

[14] H. K. Versteeg and W. Malalasekera, An Introduction to Computational Fluid Dynamics: The Finite Volume Method Longman Scientific and Technical, 1995.

[15] J. H. Ferziger and M. Peric', Computational Methods for Fluid Dynamics Springer, Berlin, 1996.

[16] Henk Kaarle Versteeg, Weeratunge Malalasekera (2007). An Introduction to Computational Fluid Dynamics: The Finite Volume Method.

[17] Bauer, Bob. 2005. Sudden, Large Waves a Lake Michigan Danger. Illinois State Geological Survey

[18] Gauthier, Roger and Manninen, Christine. 1999. Living with the Lakes. US Army Corps of Engineers and Great Lakes Commission, 1999. pgs. 15-17.

[19] Arthur McGarr and Robert C. Vorhis, 1968, Seismic seiches from the March 1964 Alaska earthquake, USGS Report, https://doi.org/10.3133/pp544E

[20] Niedda, Marcello Greppi, Mauro. (2007). Tidal, seiche and wind dynamics in a small lagoon in the Mediterranean Sea. Estuarine, Coastal and Shelf Science. 74. 21-30. 10.1016/j.ecss.2007.03.022.

[21] ong-Ye Wu, En-Yun Hsu, Robert L. Street, Experimental study of nonlinear wave - wave interaction and white-cap dissipation of wind-generated waves, Dynamics of Atmospheres and Oceans, Volume 3, Issue 1, 1979, Pages 55-78, ISSN 0377-0265, https://doi.org/10.1016/0377-0265(79)90037-X.

[22] Grimshaw, R. (1975). Nonlinear Internal Gravity Waves and Their Interaction with the Mean Wind, Journal of Atmospheric Sciences, 32(9), 1779-1793

[23] Young, I. R., and G. Ph. Van Vledder. "A Review of the Central Role of Nonlinear Interactions in Wind-Wave Evolution." Philosophical Transactions: Physical Sciences and Engineering, vol. 342, no. 1666, The Royal Society, 1993, pp. 505-24, http://www.jstor.org/stable/54171. 